\title{
The Prediction Research of Population Density Based on Deep Learning in Grain Stored Insects
}

\author{
Wu Jian-Jun ${ }^{1}$, Dang Hao ${ }^{1}$, Li Miao ${ }^{1}$, Sun Fu-Yan ${ }^{1}$, Zhu Yu-Hua ${ }^{1}$, Zhen Tong ${ }^{1,2}$ \\ and Zou Bing-Qiang ${ }^{3}$ \\ ${ }^{1}$ School of Information Science and Engineering, Henan University of Technology, \\ Zhengzhou 450001, China \\ ${ }^{2}$ Collaborative Innovation Center for Modern Grain Circulation and Safety, \\ Nanjing, 210023, China \\ ${ }^{3}$ Shandong College of Information Technology, Software Department Weifang \\ 261061, China \\ popp_ly@163.com \\ Abstract
}

\begin{abstract}
Precision of pests, in stored grain insect population density, has been a hot and difficult research in pest detection and control system. The accuracy of prediction of pest density will directly affect to warehouse grain temperature and the food quality etc. In order to improve the accuracy, the paper which wising the depth study method, established an insects density prediction mode with the depth of the beter network as the core. The model is applied to the algorithm of deep learning predictive control. According to the temperature and humidity of the graicobiained from the actual measurement and the initial density of the pest, we predicted the pest density. Simulation results show that the root mean square error is smallbetween the predictive value and actual value, high prediction accuracy. The deep learning atgonith $n$ is applied to the population density of pests is effective.
\end{abstract}

Keywords: deeplearning, population density, deep confidence network, predictive and control Introduction

\section{Introduction}

The harm of the pest in grain storage is a major technical problem that have an influence on the quality and safety of grain storage at present. The bottleneck problem is pest population ensity detection and prediction. And the population density of grain stored insects is a crucial measure to simulate and verify pest occurrence and growth trend. Grain reserves cycle in the United States and Japan generally maintained at 1-2 years, so 1 thould take measures when per ton of food exists five head of pests. However, due to different national conditions, China's grain reserves cycle is in 3-5 years, in addition to the number of pests in a certain period increase with a doubling of power series, so the decision standard of dealing with insect pest is basically per ton of exits 2 head pests.

Traditional prediction of pest population density basically is to rely on statistics, but its large workload and the high labor intensity seriously affect the efficiency and accuracy of forecast. The article take the Deep Learning (Deep Learning, DL) modeling method can make up for the inadequacy. Deep Learning is a branch of the machine learning. It also is an extension of neural network, and its main character is to obtain the expression of different abstract layer for original data by multi-level learning, and then improve the accuracy of classification and prediction. The layered idea of Deep Learning model 
achieve hierarchical expressed for input information, which greatly improve the timeliness and accuracy to the whole operation.

\section{The Basic Theory of Deep Learning Algorithm}

Deep Learning is a new field of machine Learning. It is a mathematical model to simulate human brain's analysis and study that based on neural network, which imitate the brain mechanisms to identify the target and sense information [1]. The traditional neural network algorithm have two main ways to select features, one is manual and it is very arduous, anther is heuristic and it mainly depends on professional knowledge. The superior level of selection mainly is association with experience and luck, and it also needs a lot of time to algorithm adjust. But Deep Learning algorithm involved in the study of feature selection in the absence of people. The representative results are Auto Encoder that was lunched by Hinton in $2006^{[2]}$, which made amazing progress on the handwritten. digit recognition.

Deep Learning mainly have three characteristics: 1) the hierarchical structure. It emphasized the depth of model structure;2) Deep Learning clearly highlight the characteristics of the importance of self-learning, its cognitive process is step by step and abstracted gradually;3) its train mechanism is different from the training of the neural network. Deep Learning hierarchical model (Figure 1) is similar with neural network and the system mainly includes input layer, hidden ayer (many layers) and output layer. Compared with neural network, the number of hidden layer is more, which show the strong ability of learning essential charactenistics of datacconcentrations from a few samples, and greatly improve the generalization ability and computing speed of complex classification problems.

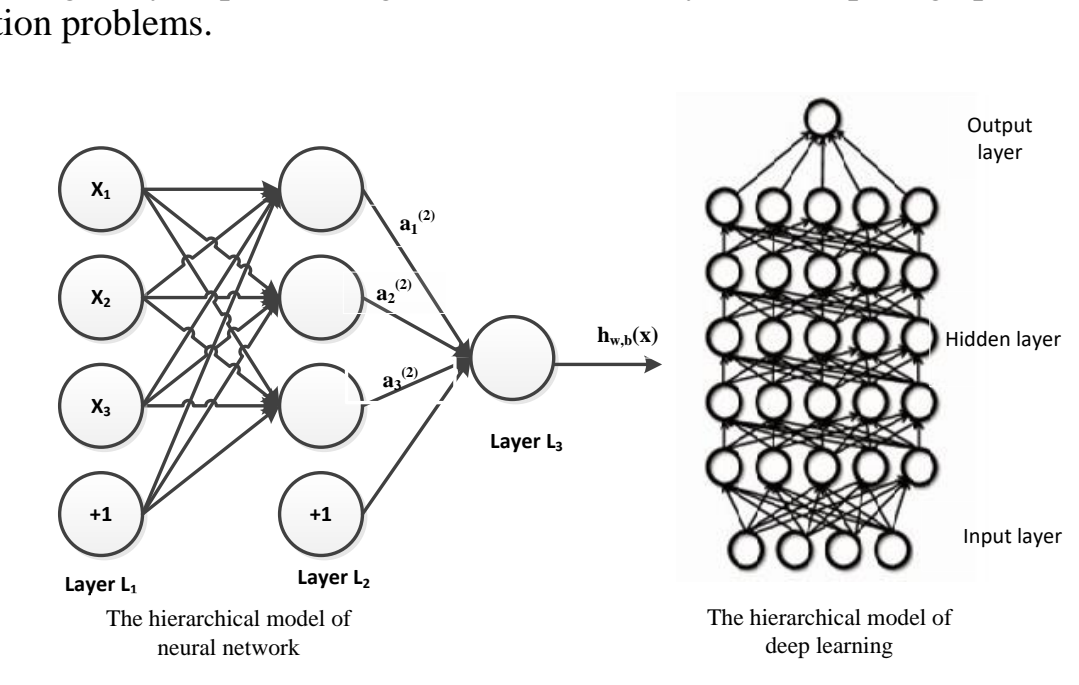

\section{Figure 1. The Hierarchical Model of Neural Network (left) and the} Hierarchical Model of Deep Learning (right)

Characteristics of self-learning is a chief breakthrough of Deep Learning, through Learning a Deep nonlinear network structure to realize the complex function approximation, which can use less parameters present complex function (Figure 2). Formed by combining low-level features more abstract high-level layers to express category or features, and transform the feature expression of the sample in the original space into a new feature space by feature conversion, so that the classification or prediction is more easily. 


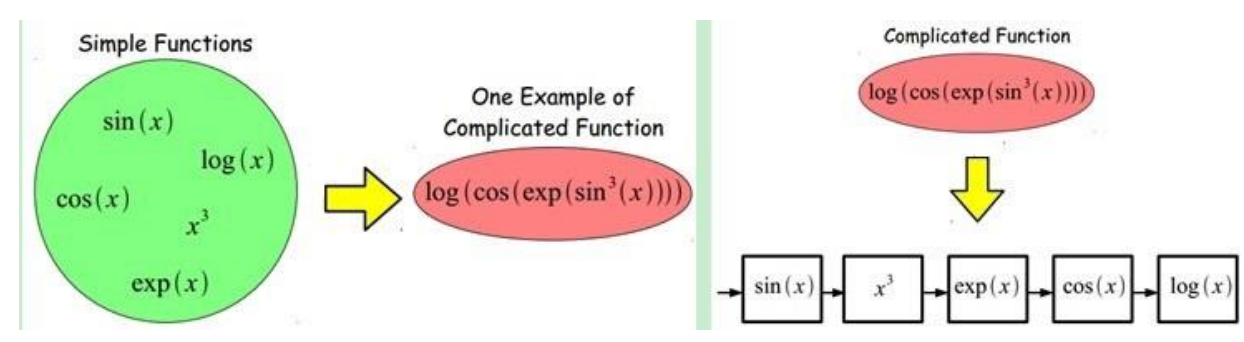

Figure 2. Complex Function Model

Deep Learning and neural network is adopted very different training mechanism. Back propagation is used in traditional neural network. Simply, using iterative algorithm to train the network with random initialization and calculate the output of the current network, then according to the difference between the current outputs and label to change the parameters of each layer until convergence. .However, deep learning is a layer-wise training mechanism entirely. The reason is that if the mechanism of back propagation is adopted, for a deep network (layer 7 above), the residual has become tor small when spread to the layer so that so-called gradient diffusion is presented.

\section{The Research Insect Population Density P ediction Moder}

\subsection{The Prediction Identification Model Design of Insect Population Density}

Deep Belief Network (DBN) within deep learning is Introduced to establish the prediction identification model of pest population density based on DBN algorithm. Pest densities basic recognition model structure diagram is hown in Figure 3. In Figure 3, x (t) is four dimensional input vector of the benchmak system for the pest population density, i.e. temperature, humidity, moisture and pest initial density; $\mathrm{y}(\mathrm{t})$ is the system output, the output is population density (head/t); $\mathrm{y}^{*}(\mathrm{t})$ is identification output of the forecast of population density by the encoding algorithm model; Otherwise, it obtains error coefficient by subtracting between $y^{*}(t)$ and $y(t)$, if error exceed the scope of regulation, the training begin unt 1 within the prescribed scope, by contrary, the DBN algorithm is applied to the pest population density basic recognition model, according to the different input parameters to predict the population density corresponding to the output.

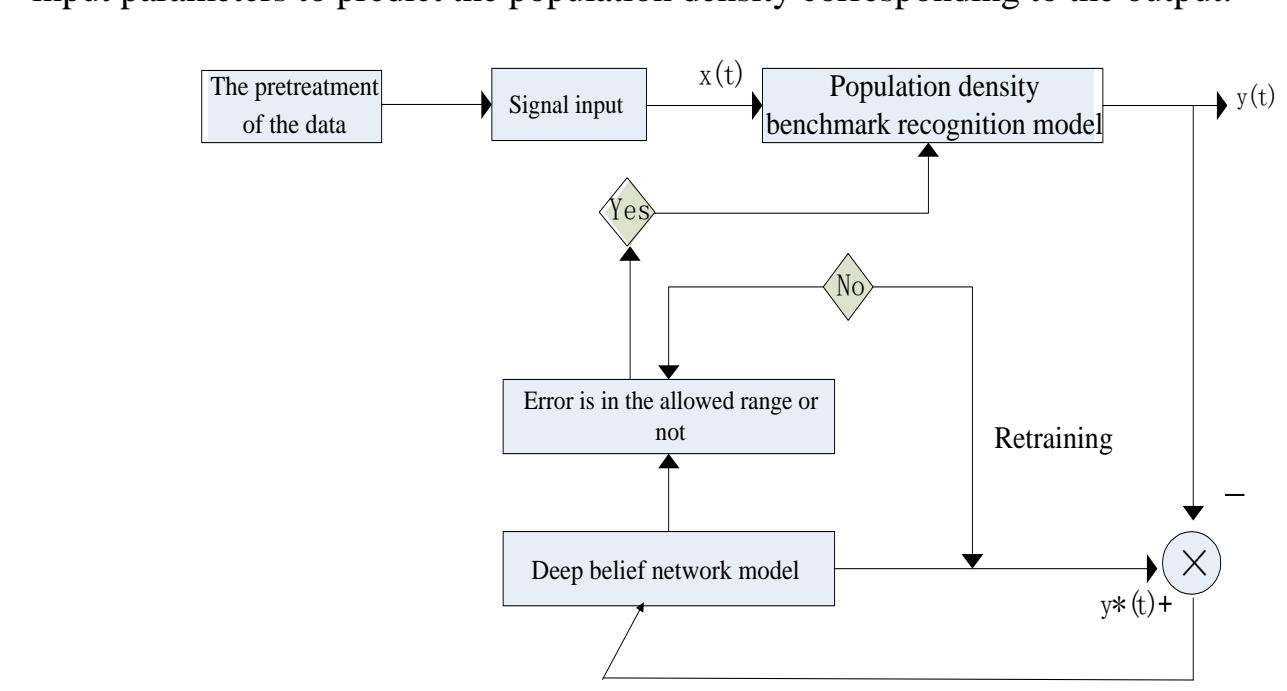

Figure 3. Insect Population Density Benchmark Recognition Model Structure 


\subsection{The Prediction Identification Model Training Algorithm Based on the Pest Population Density}

DBN is the Bayesian Probability Generation Model, which is consisted of multiple Restricted Boltzmann Machines layer[3]. The two layers on above is the non-directional symmetric connections, the layer on below obtain directional connection from a layer of top, the status of bottom unit is visible input data vector .DBN is composed of several structural unit stack, as shown in Figure 4, the structural unit network is limited as a visual layer and a hidden layer, the hidden layer units are trained to catch higher order data correlation that expressed in the visual layer. In stack, the number of visual layer neuron in RBM unit is equal to the number of previous hidden layer neuron in RBM. According to deep learning mechanism, inputting the first layer sample training units, and training the second layer in the second RBM model make use of its output, then stack RBM model make use of increase layers to improve the model efficiency. In the process of unsupervised beforehand training, the reconstruction of input is realized from the top layer decode state to the layer units below when DBN coding is inputted to the top layer RBM [4].

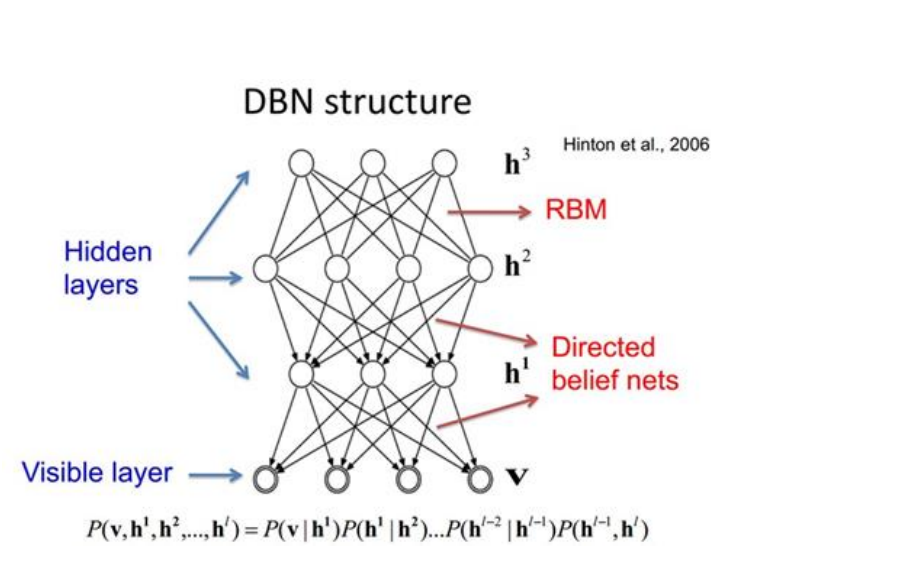

\section{Figure 4. The Structure of DBN}

RBM is undirected graph probability model based on energy[5], the joint probability distribution that defined by the energy function of input $x$ and hidden variable $h$ is

$$
\text { Q } \mathrm{p}(\mathrm{x}, \mathrm{h})=\frac{e^{- \text {energy }(x, h)}}{Z}
$$

The normalization onstant $\mathrm{Z}$ in (1) is called as partition function[6]. Observed marginal probabilitydestroution of the input $\mathrm{x}$ is

$$
\mathrm{p}(\mathrm{x})=\sum_{\mathrm{h}} \mathrm{p}(\mathrm{x}, \mathrm{h})=\sum_{\mathrm{h}} \frac{\mathrm{e}^{- \text {energy }(\mathrm{x}, \mathrm{h})}}{\mathrm{z}}
$$

The introduction of free variables can change the (2) into

$$
\mathrm{p}(\mathrm{x})=\frac{\mathrm{e}^{- \text {freeEnergy }(\mathrm{x})}}{\mathrm{z}}
$$

The $z=\sum_{x} e^{-f r e e E n e r g y(x)}$ in (3) is freeEnergy $(x)=-\log \sum_{h} e^{-\operatorname{energy}(x, h)}(4)$ Introducing $\theta$ expresses the parameter of model, the (3) is figured out logarithm and
derivative into[7]

$$
\frac{\varphi \log p(\mathrm{x})}{\varphi \theta}=-\frac{\varphi \text { freeEnergy }(\mathrm{x})}{\varphi \theta}+\frac{1}{\mathrm{Z}} \sum_{\mathrm{x}}-\mathrm{e}^{- \text {freeEnergy }(\mathrm{x})} \frac{\varphi \text { freeEnergy }(\tilde{\mathrm{x}})}{\varphi \theta}
$$

It is difficult that figured out partition function ${ }^{[8]}$, therefore to training it with the approximation of $\operatorname{logarithmic}$ likelihood gradient $\frac{\varphi \log \mathrm{P}(\mathrm{x})}{\varphi \theta}$, using obey the free energy gradient of the data distribution sample $\mathrm{x} \sim \mathrm{p}(\mathrm{x})$ and the model distribution sample $\tilde{\mathrm{x}} \sim \mathrm{p}(\tilde{\mathrm{x}})$ to define the rules of the model parameters update

$$
\mathrm{E}_{\widehat{\mathrm{p}}}\left[\frac{\varphi \log \mathrm{P}(\mathrm{x})}{\varphi \theta}\right]=-\mathrm{E}_{\widehat{\mathrm{p}}}\left[\frac{\varphi \text { freeEnergy }(\mathrm{x})}{\varphi \theta}\right]+\mathrm{E}_{\mathrm{p}}\left[\frac{\varphi \text { freeEnergy }(\widetilde{\mathrm{x}})}{\varphi \theta}\right]
$$


Among them: $\hat{\mathrm{p}}$ is the empirical probability distribution of training data set, and $\mathrm{p}$ is a model probability distribution, $\mathrm{E}_{p}$ and $\mathrm{E}_{\hat{p}}$ is the expectations under the corresponding

probability distributions. the first part is easy to calculate in (6) [9], usually replaced with the average approximation of training sample; The second part contains samples collected

from the model p sampling, which usually use some approximate sample to replaced algorithm[10].

It can use the approximate maximum likelihood stochastic gradient descent to train BRM algorithm, which usually adopt the Monte Carlo Markov chain (Monte - Carlo Markov chain, MCMC) method to get the sample models [11].

The training process of DBN is as follows:

a) The first layer be regarded as an initial input model, using unsupervised training method, made the original input reconstruction error diminish;

b) The output of hidden unit of DBN model be convinced that the input of another

layer [11];

c) Iterated initialization parameters of each layer according to b);

d) Using the output of the last hidden layer as input, and inflict a supervised layer (usually the output layer), and initialize the layer parameters [12];

e) Adjust the all parameters of DBN according to the supervision cyterion, which compose DBN.

\section{The Prediction and Result of Population Density for Grain Stored Insects}

\subsection{The Collection of Training Samples}

All training datas are from Qingyuan state grain reserve in the Baoding of Hebei province. The grain and population of insect is egard as research object and benchmark within experiment. A total of 30 groups of data are collected by stick insect acquisition methods in three mouths (o1 July, August and September in 2014. The storage of grain house is 5700 tons and insect of grain is Liposcelis entomophily and granary ventilate by negative pressure and spraying $50 \mathrm{~kg}$ of inert powder. All collected datas is training data of deep belief network. Otherwise, select 15 sets as the validation data, and finally use the rest of the 15 sets of datas to test the prediction performance of the network. Part of the experiment data are shown in Table 1 .

Table 1. All the Experiment Data

\begin{tabular}{|c|c|c|c|c|c|}
\hline Time & cal & $\begin{array}{l}\text { Quantity/H } \\
\text { ead }\end{array}$ & $\begin{array}{c}\text { Temperature/ } \\
{ }^{\circ} \mathrm{C}\end{array}$ & $\underset{\%}{\text { Humidity/ }}$ & $\begin{array}{c}\text { Initial } \\
\text { density/Head/ton }\end{array}$ \\
\hline & 1 & 380 & 31.5 & 62.5 & \multirow{5}{*}{0.23} \\
\hline & 2 & 420 & 32.0 & 67.4 & \\
\hline & 3 & 440 & 32.6 & 68.0 & \\
\hline & 4 & 520 & 34.2 & 63.2 & \\
\hline & 5 & 490 & 33.7 & 67.3 & \\
\hline \multirow{5}{*}{ May } & 1 & 430 & 33.4 & 71.2 & \multirow{5}{*}{0.34} \\
\hline & 2 & 510 & 35.6 & 73.7 & \\
\hline & 3 & 470 & 32.9 & 68.3 & \\
\hline & 4 & 420 & 33.3 & 69.5 & \\
\hline & 5 & 440 & 31.9 & 59.7 & \\
\hline June & 1 & 510 & 34.3 & 66.4 & 0.21 \\
\hline
\end{tabular}




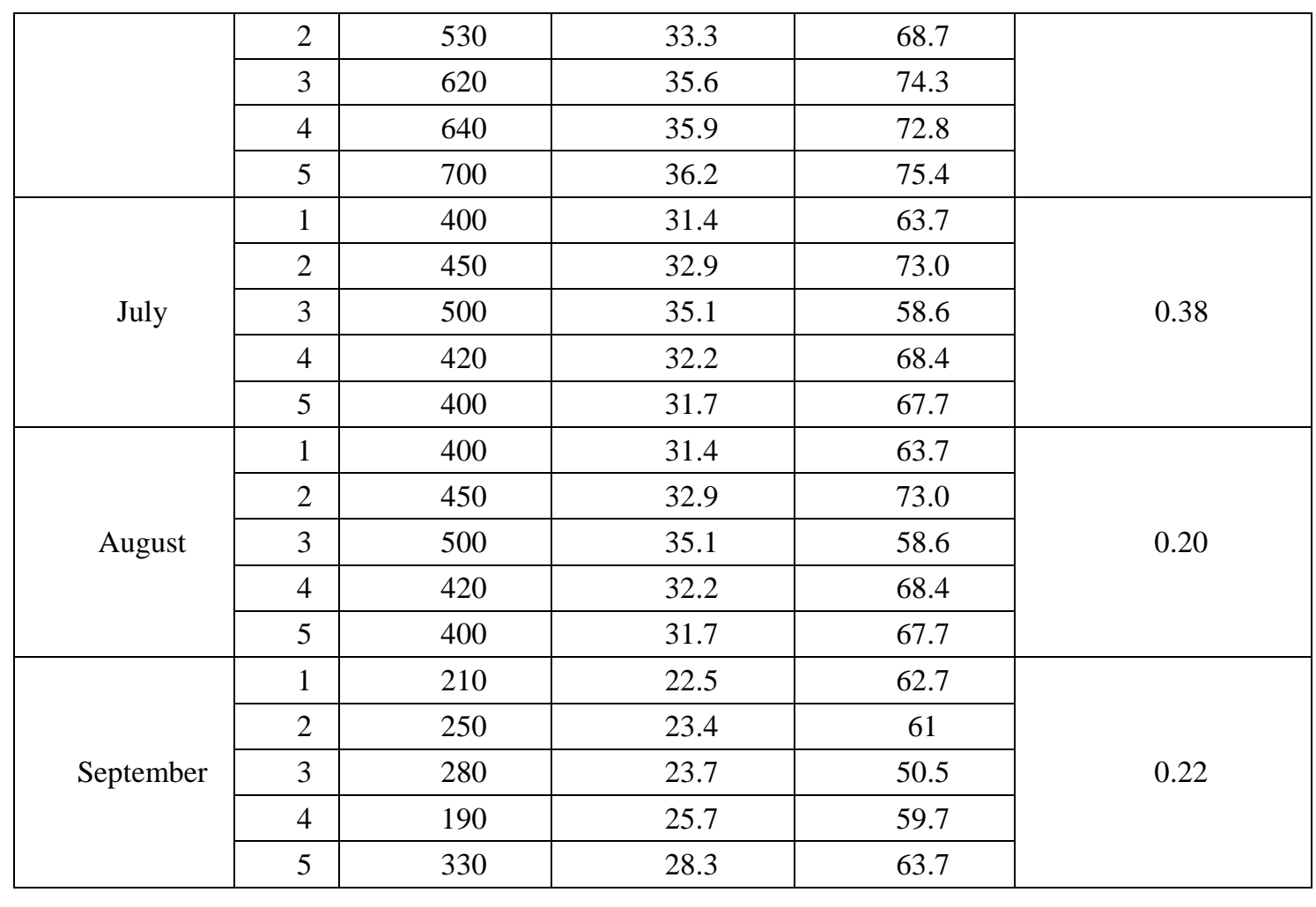

\subsection{The Pretreatment of the Data}

The prediction parameters of pest population density include the initial density of the pest, grain temperature and humidity [14], as a result of these predictors are different data types and order of magnitude, therefore, the original data need to be normalization processing .This article uses the method of maximum minimum to normalized processing of original data, the data atrer processing is in between 0 and 1 , it is conducive to training algorithm. Computational expressions are as follows [15]:

In the type, $\hat{\mathrm{X}}$ is the processing of data, $\mathrm{X}$ is as the original data, $\mathrm{X}_{\max }$ and $\mathrm{X}_{\min }$ are the maximum and the minimun of the original data respectively [16].

\subsection{The Experimental Results and Analysis}

Based on DBN pests in population density prediction simulation results are shown in Figure 5 below. Through artificial screening of actual density value is shown in Figure 6 . Can be seen from the diagram of the training sample after 7 that predicted and actual values is similar, the accuracy reached $75 \%$ to $80 \%$, and the sample size within 7 pred cted value and actual value is large, basic continued accuracy within $40 \%$, part of the point even only about $20 \%$. And the density of prediction accuracy is around 1.5 per ton. Because when the population density is lower than the identification model of the kernel function (1.5), the logarithmic of temperature $\log t$ and the logarithm of population density $\log d$ is a nonlinear relationship, the model identification error is large; And when the density is greater than 1.5 per ton, it is happened to be in line with the identification model of the kernel function, the logarithm of the temperature is a linear relationship with the logarithm of population density, relatively easy to measure precise values. 


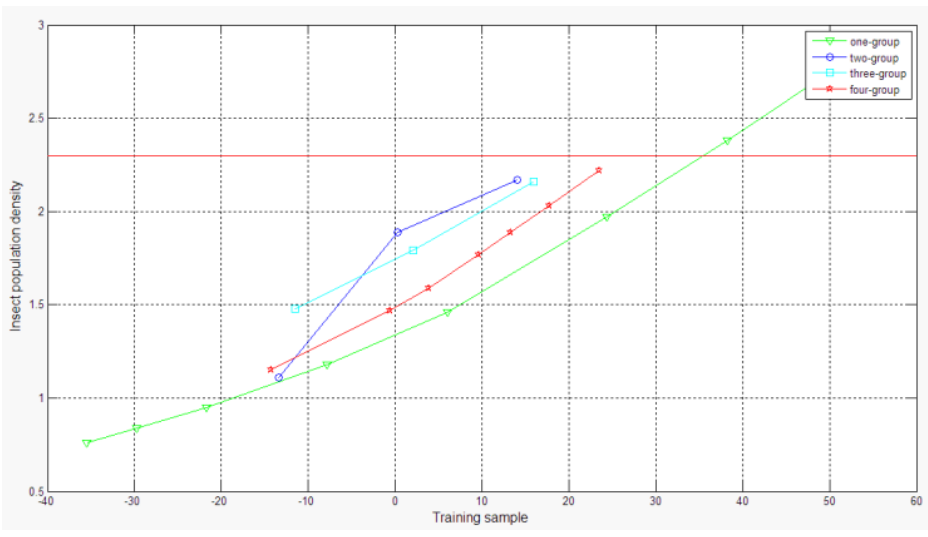

Figure 5. The Prediction Result of Insect Population Density

\section{Conclusion}

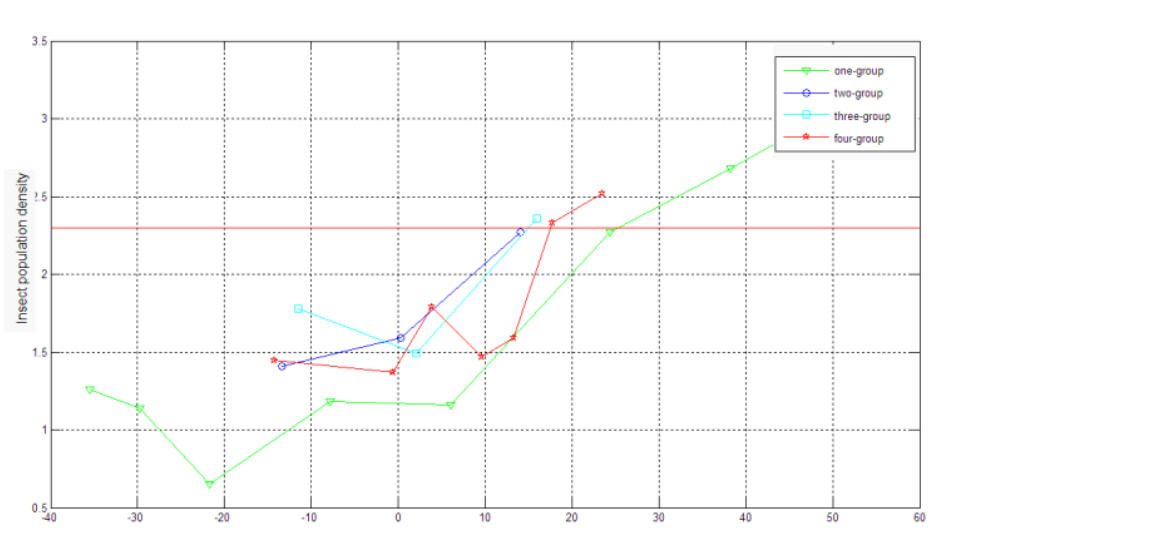

Figure 6. The Real Result oOnsect Population Density

Liposcelis entomophila is regard as the research object in the article Based, using the deep learning algorithms to established pests population density prediction system identification model with DBSY, and appliing the model to study in the depth of the pest in predictive control algofith 9 [9],. Through the advance gathering sample data to identify model of training, tratming after the model can identify the input data (temperature, humidity, and the initial density) of insect pests and non-linear relation between the output data (popuration density), according to the input data to predict the population density. The simulation results show that population density prediction model based on DBN algorthm has better prediction precision. Through the training data mining and analysis the pests in population density has an important guiding significance to the dejelopment of prediction technology.

\section{Acknowledgements}

Sincerely thank the Project supported by the Special Fund for Grain scientific, Research in the Public Interest (Grant No: 201413003 ), and Key scientific research project of Henan Province (Grant No:280090 ), and Henan University of Technology, Doctor Fund(Grant No:150575 ), and Open fund of key Laboratory of Grain Information Processing and Control(under Grant No.KFJJ-2015-103). 


\section{References}

[1] H.-L. Hu, W. Wei and M.-N. Hu, "Principles and practices of deep learning", Information Technology, vol. 45, no. 2, (2015), pp.175-177.

[2] G. E. Hinton and S. Osindero, "The Y W.A Fast Learning Algorithm for Deep Belief Nets", NeuralComputation, vol.18, no. 7, (2006), pp.1527 - 1554.

[3] C. Xia, "Prediction of Moisture Content of Wood Based on Deep Learning", Journal Of Hangzhou Dianzi University (Natural Sciences), vol.35, no. 1, (2015), pp31-35.

[4] G. Liu, "Reasearch into speech Reconition Based on Deep Learning", BeiJing, Beijing University of Posts and Telecommunications, (2014).

[5] W. Li, "The research and application of deep learning in image recognition", Wu Han, Wuhan University of Technology, (2014).

[6] H. La Rochelle, Y. Bengio, J. Lou Radour, "Exploring strategies for training deep neural networks", Journal of Machine Learning Research, vol. 10, no. 12, (2009), pp. 1-40.

[7] G. Taylor, L. Sigal and D. J. Fleet, "Dynamical binary latent variable models for 3D human pose tracking", Proc of IEEE Conference on Computer Vision and Pattern Recognition, (2010), pp. 631-638.

[8] K. Jar Rett, K. Kavukcuoglu and M. Ranzato, "What is the best multi-stage architecture for object recognition?", Proc of the 12th International Conference on Computer Vision, (2009), pp. 2146-2153

[9] H. Lee, R. Grosse and R. Ranganath, "Convolutional deep belief networks for scalable unsupervised learning of hierarchical representations", Proc of the 26th International Conference on Machine Learning. New York: ACM Press, (2009), pp. 609-616.
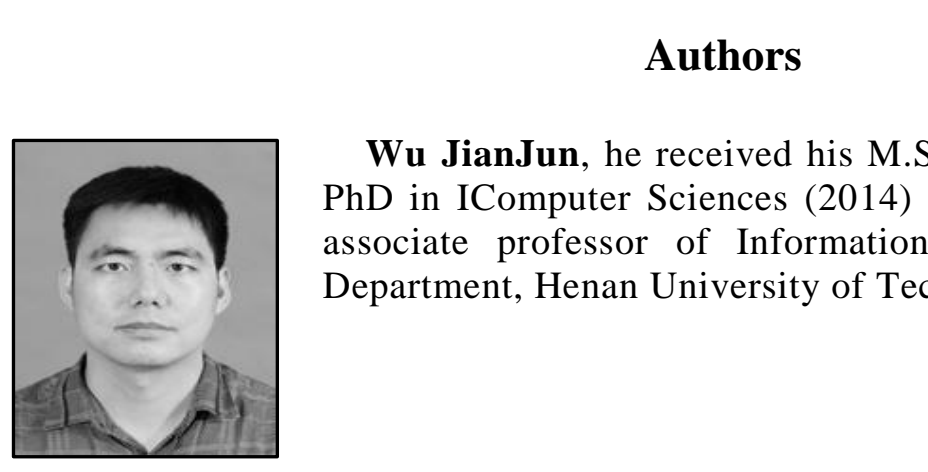

Wu JianJun, he received his M.Sc in Computers (2008) and $\mathrm{PhD}$ in IComputer Sciences (2014) form University. Now he is associate professor of Information Science and Engineering Department, Henan University ofTechnology Since 1999.

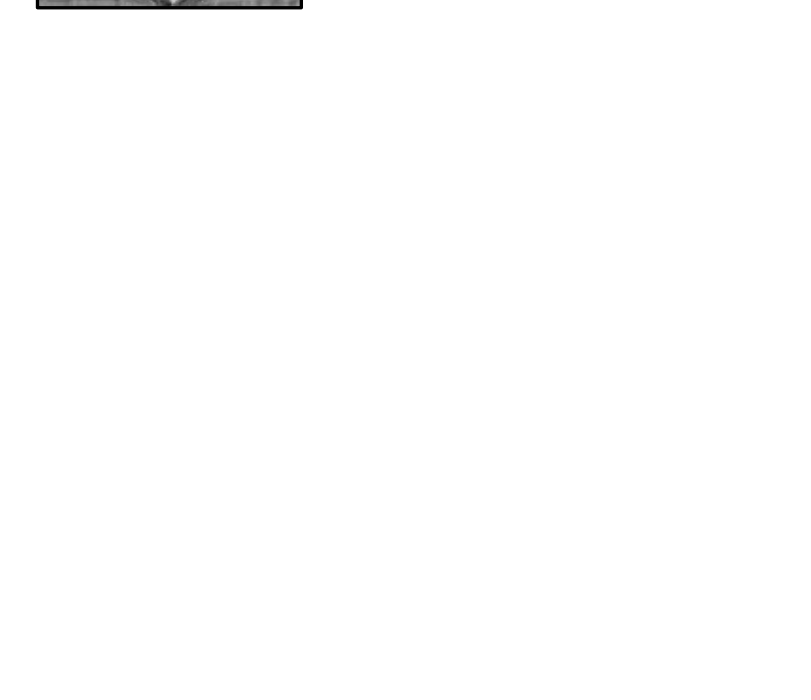

